ANNALES

POLONICI MATHEMATICI

$86.2(2005)$

\title{
A counterexample to the regularity of the degenerate complex Monge-Ampère equation
}

\author{
by SzYMON PLiś (Kraków)
}

\begin{abstract}
We modify an example due to X.-J. Wang and obtain some counterexamples to the regularity of the degenerate complex Monge-Ampère equation on a ball in $\mathbb{C}^{n}$ and on the projective space $\mathbb{P}^{n}$.
\end{abstract}

1. Introduction. The Monge-Ampère operator of a smooth plurisubharmonic function $u$ is given by

$$
\left(d d^{c} u\right)^{n}=4^{n} n ! \operatorname{det}\left(u_{i \bar{j}}\right) d \mathcal{L}, \quad \text { where } \quad u_{i \bar{j}}=\frac{\partial^{2} u}{\partial z_{i} \partial \bar{z}_{j}}
$$

and $\mathcal{L}$ is the $2 n$-dimensional Lebesgue measure. For an arbitrary continuous plurisubharmonic function $u$ one can define $\left(d d^{c} u\right)^{n}$ as a regular Borel measure. Let $\Omega$ be a strictly pseudoconvex domain in $\mathbb{C}^{n}$ (throughout the note we always assume $n \geq 2$ ). Then for any nonnegative $f$ which is continuous in $\Omega$ and for $\varphi$ continuous on $\partial \Omega$ the Dirichlet problem

$$
\left\{\begin{array}{l}
u \in \operatorname{PSH}(\Omega) \cap \mathcal{C}(\bar{\Omega}), \\
\left(d d^{c} u\right)^{n}=f d \mathcal{L} \quad \text { in } \Omega, \\
u=\varphi \text { on } \partial \Omega,
\end{array}\right.
$$

has a unique solution (see $[\mathrm{B}-\mathrm{T}]$ ).

Below we list some regularity results for solutions of (1.1):

(1) $\partial \Omega \in \mathcal{C}^{\infty}, \varphi \in \mathcal{C}^{\infty}(\partial \Omega), f \in \mathcal{C}^{\infty}(\bar{\Omega}), f>0 \Rightarrow u \in \mathcal{C}^{\infty}(\bar{\Omega})$ (Caffarelli, Kohn, Nirenberg and Spruck [C-K-N-S]);

(2) $\partial \Omega \in \mathcal{C}^{3,1}, \varphi \in \mathcal{C}^{3,1}(\partial \Omega), f^{1 / n} \in \mathcal{C}^{1,1}(\bar{\Omega}), f \geq 0 \Rightarrow u \in \mathcal{C}^{1,1}(\bar{\Omega})$ (Krylov [Kr1, Kr2]).

There are analogous regularity theorems for the real Monge-Ampère equations on a strongly convex domain $\Omega$ in $\mathbb{R}^{n}$ (see $[\mathrm{C}-\mathrm{K}-\mathrm{N}]$ and $[\mathrm{G}-\mathrm{T}-\mathrm{W}]$ ). In a forthcoming paper about the degenerate Monge-Ampère equation on

2000 Mathematics Subject Classification: 32W20, 35J70.

Key words and phrases: complex Monge-Ampère operator. 
strongly pseudo-convex domains in $\mathbb{C}^{n}$, the author proves the following complex version of a result from $[\mathrm{G}-\mathrm{T}-\mathrm{W}]$ :

(3) $\partial \Omega \in \mathcal{C}^{3,1}, \varphi \in \mathcal{C}^{3,1}(\partial \Omega), f^{1 /(n-1)} \in \mathcal{C}^{1,1}(\bar{\Omega}), f \geq 0 \Rightarrow u$ is almost $\mathcal{C}^{1,1}$ (i.e. mixed complex derivatives $u_{i \bar{j}}$ are bounded).

Similar results are true for Kähler manifolds. Let $M$ be a compact Kähler manifold of complex dimension $n$, with the Kähler form $\omega$. We will say that a continuous function $\phi$ on $M$ is admissible if $d d^{c} \phi+\omega \geq 0$. For any nonnegative $f$ which is continuous on $M$ and satisfies the necessary condition

$$
\int_{M} f \omega^{n}=\int_{M} \omega^{n}
$$

the Monge-Ampère equation

$$
\left\{\begin{array}{l}
\phi \text { is admissible, } \\
\left(d d^{c} \phi+\omega\right)^{n}=f \omega^{n} \quad \text { in } M,
\end{array}\right.
$$

has a unique (up to a constant) continuous solution (see [K1, K2, B3]).

We have the following results about regularity of (1.3):

(4) $f \in \mathcal{C}^{\infty}(M), f>0 \Rightarrow \phi \in \mathcal{C}^{\infty}$ (Yau $\left.[\mathrm{Y}]\right)$;

(5) $f^{1 /(n-1)} \in \mathcal{C}^{1,1}(M), f \geq 0 \Rightarrow \phi$ is almost $\mathcal{C}^{1,1}$ (Błocki [B2]).

In [W] Wang proved that the exponent $1 /(n-1)$ is optimal for analogous results in the real case. In this paper we show that in (3) and (5) the constant $1 /(n-1)$ is also optimal. Our examples are very similar to Example 3 in [W] but in the proof of Lemma 2.1 below, although the idea is also similar to [W], at some point we proceed quite differently than in the real case.

2. Examples. Let

$$
f(z)=A \eta\left(\frac{\left|z_{n}\right|}{\left|z^{\prime}\right|^{\alpha}}\right)\left|z^{\prime}\right|^{\beta}, \quad\left(z_{1}, \ldots, z_{n-1}, z_{n}\right)=\left(z^{\prime}, z_{n}\right)=z \in \mathbb{C}^{n}
$$

where $\alpha, \beta>2, A>0$ and

$$
\eta(t)= \begin{cases}e^{-1 /\left(1-t^{2}\right)}, & |t|<1, \\ 0, & |t| \geq 1 .\end{cases}
$$

Let $B$ be the unit ball in $\mathbb{C}^{n}$.

We need the following lemma:

Lemma 2.1. Let $u \in \operatorname{PSH} \cap \mathcal{C}(B)$ be such that $\left.u\right|_{\{0\} \times \mathbb{C} \cap B}$ is not constant,

$$
\left(d d^{c} u\right)^{n}=f d \mathcal{L} \quad \text { in } B
$$

where $f$ is given by (2.1) and

$$
u\left(z^{\prime}, z_{n}\right)=u\left(w^{\prime}, w_{n}\right) \quad \text { if }\left|z^{\prime}\right|=\left|w^{\prime}\right| \text { and }\left|z_{n}\right|=\left|w_{n}\right| .
$$


Then

$$
u\left(0, \varepsilon_{k}\right)-u(0) \geq C \varepsilon_{k}^{(2 \alpha+2(n-1)+\beta) / n \alpha}
$$

for some sequence $\varepsilon_{k} \searrow 0$ and $C>0$ depending only on $\alpha, \beta$ and $n$. In particular, if $\beta<2(n-1)(\alpha-1)$, then $u$ is not $\mathcal{C}^{1, a}$ smooth for some $a<1$.

Proof. 1. By the maximum principle, if $\left|z^{\prime}\right| \leq\left|w^{\prime}\right|$ and $\left|z_{n}\right| \leq\left|w_{n}\right|$, then $u\left(z^{\prime}, z_{n}\right) \leq u\left(w^{\prime}, w_{n}\right)$.

2. Let $h(x)=u\left(0, e^{x}\right)$ for $x \in[-\infty, 0)$. Then $h$ is convex and reaches its strict minimum at $x=-\infty$. Indeed, suppose that $m=\sup \{x: h(x)=$ $h(-\infty)\} \neq-\infty$. There are $\mu>0$ such that

$$
h(m+2 \mu)<u\left(e^{(m-2 \mu) / \alpha}, 0, \ldots, 0, e^{m-2 \mu}\right)
$$

and an affine function $l$ such that $l(m-\mu)=h(m-\mu)$ and $l(m+\mu)=$ $h(m+\mu)$. So

$$
\overline{\left\{u\left(z^{\prime}, z_{n}\right)<l\left(\log \left|z_{n}\right|\right)\right\}} \subset V=\left\{\max \left\{e^{m-2 \mu},\left|z^{\prime}\right|^{\alpha}\right\}<\left|z_{n}\right|<e^{m+2 \mu}\right\} .
$$

But $u$ is maximal in $V$, so this is impossible.

3. There is a sequence $\varepsilon_{k} \searrow 0$ such that $u\left(z^{\prime}, z_{n}\right)=u\left(0, \varepsilon_{k}\right)$ for $\left|z^{\prime}\right|^{\alpha}<$ $\left|z_{n}\right|=\varepsilon_{k}$. Indeed, there is a sequence $\varepsilon_{k} \searrow 0$ such that $h$ is strictly convex in $\varepsilon_{k}$ for $k \in \mathbb{N}$, i.e. there is an affine function $l$ such that $\{l(x)<h(x)\}=$ $[-\infty, 0) \backslash\left\{\varepsilon_{k}\right\}$. From continuity and maximality of $u$ (in int $\{f=0\}$ ) it is clear that for every small $s>0$ we have $l\left(\log \left|z_{n}\right|\right)+s \geq u(z)$ for $z=\left(z^{\prime}, z_{n}\right)$ whenever $\left|z_{n}\right|=\varepsilon_{k}$ and $\left|z^{\prime}\right|^{\alpha}<\left|z_{n}\right|$.

4. Let $\varepsilon=\varepsilon_{k}$ and $\lambda=\varepsilon^{1 / \alpha}$. Let $T$ denote the transformation $\left(w^{\prime}, w_{n}\right)=$ $T\left(z^{\prime}, z_{n}\right)=\left(z^{\prime} / \lambda, z_{n} / \varepsilon\right)$, and let

$$
v\left(w^{\prime}, w_{n}\right)=\frac{u\left(\lambda w^{\prime}, \varepsilon w_{n}\right)-u(0, \varepsilon)}{\left(\varepsilon \lambda^{n-1}\right)^{2 / n}} .
$$

Then $v<0$ in $B$ and $\left(d d^{c} v\right)^{n}=f \circ T^{-1}$.

5. Let $\psi=|z|^{2}-1$. Then $\psi \in \operatorname{PSH}^{-}(B), \lim _{z \rightarrow \partial B} \psi=0$ and $\psi<-1 / 2$ on $\frac{1}{2} B$. So [B1, Corollary 2.3] gives us

$$
\frac{1}{2^{n}} \int_{\frac{1}{2} B}\left(d d^{c} v\right)^{n} \leq \int_{B}|\psi|^{n}\left(d d^{c} v\right)^{n} \leq\|v\|_{B}^{n} \int_{B}\left(d d^{c} \psi\right)^{n}=C_{1}\|v\|_{B}^{n} .
$$

6. Let

$$
D=\left\{z:\left|z_{n}\right|<\frac{1}{2}\left(\frac{\lambda}{8}\right)^{\alpha}, \frac{\lambda}{8}<\left|z^{\prime}\right|<\frac{\lambda}{4}\right\} .
$$

Then $D \subset T^{-1}\left(\frac{1}{2} B\right)$ and $\mathcal{L}(D) \geq C_{2} \varepsilon^{2} \lambda^{2(n-1)}$ and

$$
\min _{D} f=f\left(\frac{\lambda}{8}, 0, \ldots, 0, \frac{1}{2}\left(\frac{\lambda}{8}\right)^{\alpha}\right) \geq C_{3} \lambda^{\beta}
$$


7. Let $\widetilde{B}=\frac{1}{2} T^{-1}(B)$. We thus obtain

$$
\begin{aligned}
-v(0) & =\|v\|_{B} \geq\left(C_{4} \int_{\frac{1}{2} B}\left(d d^{c} v\right)^{n}\right)^{1 / n}=\left(\frac{C_{4}}{\varepsilon^{2} \lambda^{2(n-1)}} \int_{\widetilde{B}} f d \mathcal{L}\right)^{1 / n} \\
& \geq\left(\frac{C_{4}}{\varepsilon^{2} \lambda^{2(n-1)}} \mathcal{L}(D) \min _{D} f\right)^{1 / n} \geq C_{5} \lambda^{\beta / n}=C_{5} \varepsilon^{\beta / \alpha n} .
\end{aligned}
$$

8. We therefore conclude

$$
u(0, \varepsilon)-u(0)=\left(\varepsilon \lambda^{n-1}\right)^{2 / n} v(0) \geq C_{5} \varepsilon^{\frac{2}{n}+\frac{2(n-1)}{n \alpha}+\frac{\beta}{\alpha n}}=C_{5} \varepsilon^{\frac{2 \alpha+2(n-1)+\beta}{n \alpha}} .
$$

Now we can give our example for the unit ball in $\mathbb{C}^{n}$.

EXAMPLE 2.2. Let $f$ be given by (2.1) where $A=1, \beta=2(n-1)(\alpha-1)-1$ and $a>1 /(n-1)$. Choose $\alpha$ such that $a \geq 2 \alpha / \beta$. Then $f^{a}$ is $\mathcal{C}^{1,1}$ but the solution $u$ of (1.1) with $\Omega=B$ and $\varphi \equiv 0$ is not $\mathcal{C}^{1,1}$.

Proof. Since $f$ and $\varphi$ satisfy condition (2.2), by the uniqueness of solution, $u$ also satisfies (2.2). From Lemma 2.1 we conclude that $u$ is not $\mathcal{C}^{1,1}$.

For $\mathbb{P}^{n}$ we have the following example:

EXAMPlE 2.3. Let $\varrho:[0, \infty) \rightarrow[0,1]$ be a function of class $\mathcal{C}^{\infty}$ such that $\left.\varrho\right|_{[0,1]} \equiv 1$ and $\left.\varrho\right|_{[2, \infty)} \equiv 0$. View $\mathbb{P}^{n}$ as a Kähler manifold carrying the Fubini-Study metric $\omega=\sum g_{i j} d z_{i} \wedge d \bar{z}_{j}$. Write $\mathbb{P}^{n}=\mathbb{C}^{n} \cup \mathbb{P}^{n-1}$ where $\mathbb{P}^{n-1}$ is the hyperplane at infinity. Let $\tilde{f}: \mathbb{P}^{n} \rightarrow \mathbb{R}_{+}$be a continuous function given in local coordinates in $\mathbb{C}^{n}$ by

$$
\widetilde{f}=\frac{\varrho(|z|) f}{4^{n} n ! \operatorname{det}\left(g_{i j}\right)},
$$

where $f$ is given by (2.1), A is such that $\tilde{f}$ satisfies the necessary condition (1.2), $\beta=2(n-1)(\alpha-1)-1$ and $a>1 /(n-1)$. Choose $\alpha$ such that $a \geq 2 \alpha / \beta$. Then $\tilde{f}^{a}$ is $\mathcal{C}^{1,1}$ but the solution $\phi$ of (1.3) with $M=\mathbb{P}^{n}$ and with $\widetilde{f}$ in place of $f$ is not $\mathcal{C}^{1,1}$.

Proof. In local coordinates in $\mathbb{C}^{n}, g_{i \bar{j}}$ are given by $g_{i \bar{j}}=\left(\frac{1}{2} \log \left(1+|z|^{2}\right)\right)_{i \bar{j}}$. Let $u=\phi+\frac{1}{2} \log \left(1+|z|^{2}\right)$. Then $u$ is a continuous solution of the MongeAmpère equation

$$
\left(d d^{c} u\right)^{n}=f \varrho \quad \text { in } \mathbb{C}^{n} .
$$

Since $\phi$ is bounded, we have $\lim _{\left|z_{n}\right| \rightarrow+\infty} u=+\infty$. Then from the same argument as in the proof of Lemma 2.1 we see that the function $z_{n} \mapsto v\left(0, z_{n}\right)$ reaches its strict minimum at $z_{n}=0$. Since $\omega$ and $\widetilde{f}$ satisfy condition (2.2), by the uniqueness of solution $\phi$ satisfies (2.2) and so does $u$. From Lemma $2.1, u$ is not $\mathcal{C}^{1,1}$, so neither is $\phi$. 
Acknowledgments. I would like to express my gratitude to Professor Z. Błocki for his support and assistance.

\section{References}

[B-T] E. Bedford and B. A. Taylor, The Dirichlet problem for a complex MongeAmpère equation, Invent. Math. 37 (1976), 1-44.

[B1] Z. Błocki, Estimates for the complex Monge-Ampère operator, Bull. Polish Acad. Sci. 41 (1993), 151-157.

[B2] - Regularity of the degenerate Monge-Ampère equation on compact Kähler manifolds, Math. Z. 244 (2003), 153-161.

[B3] - Uniqueness and stability for the complex Monge-Ampère equation on compact Kähler manifolds, Indiana Univ. Math. J. 52 (2003), 1697-1701.

[C-K-N] L. Caffarelli, L. Nirenberg and J. Spruck, The Dirichlet problem for nonlinear second-order elliptic equations I: Monge-Ampère equation, Comm. Pure Appl. Math. 37 (1984), 369-402.

[C-K-N-S] L. Caffarelli, J. J. Kohn, L. Nirenberg and J. Spruck The Dirichlet problem for nonlinear second-order elliptic equations II: Complex Monge-Ampère, and uniformly elliptic, equations, ibid. 38 (1985), 209-252.

[G] P. Guan, $C^{2}$ a priori estimate for degenerate Monge-Ampère equations, Duke Math. J. 86 (1997), 323-346.

[G-T-W] P. Guan, N. S. Trudinger and X.-J. Wang, On the Dirichlet problem for degenerate Monge-Ampère equations, Acta Math. 182 (1999), 87-104.

[K1] S. Kołodziej, The complex Monge-Ampère equation, ibid. 180 (1998), 69-117.

[K2] - Stability of solutions to the complex Monge-Ampère equation on compact Kähler manifolds, Indiana Univ. Math. J. 52 (2003), 667-686.

[Kr1] N. V. Krylov, Smoothness of the payoff function for a controllable process in a domain, Izv. Akad. Nauk SSSR 53 (1989), 66-96 (in Russian); English transl.: Math. USSR-Izv. 34 (1990), 65-95.

[Kr2] - On analogues of the simplest Monge-Ampère equation, C. R. Acad. Sci. Paris 318 (1994), 321-325.

[W] X.-J. Wang, Some countrexamples to the regularity of Monge-Ampère equations, Proc. Amer. Math. Soc. 123 (1995), 841-845.

[Y] S.-T. Yau, On the Ricci curvature of a compact Kähler manifold and the complex Monge-Ampère equation, I, Comm. Pure Appl. Math. 31 (1978), $339-411$.

Institute of Mathematics

Jagiellonian University

Reymonta 4

30-059 Kraków, Poland

E-mail: Szymon.Plis@im.uj.edu.pl 